\title{
Wissen, was wir wollen - Agrarforschung widmet sich dem Konflikt um die Tierhaltung
}

\author{
Philipp von Gall ${ }^{1}$
}

Published online: 9 April 2018

(C) Bundesamt für Verbraucherschutz und Lebensmittelsicherheit (BVL) 2018

Derzeit erleben wir wahrscheinlich einen gesellschaftlichen Wertewandel im Umgang mit Tieren. Das meint auch der Wissenschaftliche Beirat des Bundesministeriums für Ernährung und Landwirtschaft (BMEL). Als Indikatoren dafür gilt der wachsende gesellschaftliche Druck auf die Politik, mehr für agrarisch genutzte Tiere zu tun und der Trend zur vegetarischen/veganen Ernährung sowie die steigende Anzahl der Medienberichte zum Thema. Doch wir wissen wenig darüber, welche Gesellschaftsgruppe welche Werte verfolgt und welche Folgen das haben könnte. Sind es vor allem junge, urbane Bevölkerungsgruppen, die den Eigennutz am billigen Schinkenbrot oder die Tradition des weihnachtlichen Gänsebratens weniger schätzen als Gerechtigkeit gegenüber Tieren und den ethischen Eigenwert tierlichen Lebens? Regieren im verstärkten Engagement für Tiere zunehmend (auch) menschliche Eigeninteressen, etwa der Schutz der natürlichen Lebensgrundlagen oder die ästhetische Abscheu gegenüber der massenhaften „Produktion“ von Tieren? Und welche Folgen hat das? Werden wir entgegen allen rechtlichen Traditionen künftig nicht-menschlichen Individuen einen juristischen Personenstatus zusprechen? Wird die heutige industrielle Tierhaltung bald gesellschaftlich so geächtet sein wie Prügelstrafen für Homosexuelle oder die Unterdrückung von Frauen am Anfang des 20. Jahrhunderts?

Das Forschungsprojekt „SocialLab - Nutztiere im Spiegel der Gesellschaft" hat sich hohe Ziele gesetzt. Es soll „Wirkzusammenhänge“ der gesellschaftlichen Akzeptanz der Tierhaltung offenlegen, die „gesellschaftliche Wahrnehmung" der Tierhaltung beschreiben und wichtige Positionen der am Konflikt um die Tierhaltung beteiligten Gruppen ausmachen. Fangen wir bei den betroffenen Tieren an: Was würden sie fordern, wenn sie

\footnotetext{
Philipp von Gall

Philipp_Gall@uni-hohenheim.de

1 Societal Transition and Agriculture, University of Hohenheim, Stuttgart, Germany
}

könnten und wer übernimmt die Aufgabe, diese Forderungen in angemessener Weise an Entscheidungsinstitutionen zu tragen? Der Staat stellt sich dieser Aufgabe der Interessenvertretung bis heute nicht und überlässt sie privaten, spendenfinanzierten Vereinen. Der Thematik tierlicher Interessen und ihrer Repräsentation geht das Projekt leider nicht nach, sondern konzentriert sich auf die Positionen der deutschen Landwirte und Verbraucher. Auch das beinhaltet große methodische Herausforderungen. Wenn so unterschiedliche Positionen wie die zur Tierhaltung konzeptualisiert werden sollen, kommt es auch auf die richtige Wortwahl an. So halten viele Tierschutzgruppen etwa den Begriff „Nutztier“ - wie im Titel des SocialLab-Projektes - für unangemessen abwertend. Um alle Positionen exakt zu erfassen, wäre es wohl am einfachsten, die Gruppen selbst sprechen zu lassen, doch das Projekt soll Positionen auswählen, zusammenfassen und ausarbeiten. Dabei sollten möglichst alle wichtigen Meinungen abgedeckt werden. Der Erfolg des SocialLab-Projektes hängt auch davon ab, wie es diese Herausforderungen meistert.

Jedenfalls wird es Zeit, die Einstellungen zur wirtschaftlichen Mensch-Tier-Beziehung besser zu verstehen. Die Kritik an der modernen Nutzung von Tieren ist nicht neu. Das 1972 eingeführte deutsche Tierschutzgesetz sollte den sozialen Konflikt um die Tierhaltung schlichten. Dies ist misslungen, der Konflikt ist seit 1972 kontinuierlich angewachsen und vielschichtig geworden. Die Tierschutzbewegung hat sich seitdem in zwei Lager geteilt: Die einen hoffen auf den Weg der kleinen Schritte hin zur „tiergerechten“ Haltung, die anderen wollen die rechtliche Wende und ein Ende der industriellen Tierhaltung. Parallel zu dieser Entwicklung wurden die Kosten pro Kilogramm produziertes Tier derart gedrückt, dass Deutschland heute in Billigproduktionsländer wie China exportiert. Doch was die Allgemeinheit darüber denkt, welche Werte und Gefühle sie mit Blick auf Tiernutzung haben, wurde in den Agrarwissenschaften bislang kaum untersucht. So ist das vom BMEL auf drei Jahre geförderte SocialLab bislang das größte seiner Art. Warum ist es einzigartig und innovativ, 
unterschiedliche Anliegen an die seit langem höchst umstrittene Tierhaltung zu analysieren? Eine Antwort darauf lautet, dass bisher angenommen wurde, die Einstellungen der Menschen zeigten sich an ihrem Kaufverhalten, die Leute 'wollten es nun mal billig'. Dass dies ein Irrtum ist, weiß die Ökonomie bereits, seit Kahnemann und Kollegen 1974 die kognitive Verzerrung beschrieben. Und lässt man den Geist der Psychologie erstmal aus der Flasche, wird es noch komplizierter. Wie auch die in diesem Band publizierten Ergebnisse zeigen, sind einige Konsument/innen darauf aus, möglichst wenig über ein Produkt zu wissen, bei dem sie moralische Probleme erwarten. Denn nur ohne ständig mit der Nase auf diese Probleme gestoßen zu werden, lässt sich der Widerspruch aushalten: Nämlich das Leid von Tieren zu missbilligen, und gleichzeitig Fleisch und andere Tierprodukte billig und ohne Skrupel einzukaufen. Deshalb glauben Menschen dem „Heile-Welt-Bild“ der Tierhaltung auf der Verpackung ihres Schnitzels, sie lassen sich von Herstellernamen wie „Weidenhof“ oder „Landglück“ beeinflussen. Sie wollen nicht wissen, ob dahinter Industriewüsten und Tierelend stehen. Und die Agrarpolitik, begleitet von der schlagkräftigen Lobbyarbeit des Deutschen Bauernverbandes, ermöglichte den Konsumenten lange Zeit diesen Wunsch. Während Käufern von Zigaretten seit Langem vorgehalten wird, (sich) zu töten, können Fleischkäufer ohne weiteres einen idyllischen Bauernhof auf den Packungen sehen. Doch gibt es Zeichen für ein Ende der kognitiven Dissonanz im Konsum. Zunehmend möchten Teile der Bevölkerung wissen, ob Tiere rechtlich ausreichend geschützt sind, oder was „mehr Tierwohl“ denn genau bedeutet. Ist zum Beispiel der in der Tierhalterbranche nunmehr diskutierte „vierte Weg“ zum Ende der betäubungslosen Ferkelkastration das, was Menschen mit dem Eigenwert des tierlichen Lebens verbinden? Oder ist die Bezeichnung dieser sogenannten kleinen Schritte als
Tierschutz oder „tiergerecht“ genau das, was Menschen seit einiger Zeit „satt haben“, setzt hier gar ein Wertewandel an? Um tierbasierte Produkte sinnstiftend mit Indikatoren zu kennzeichnen, müsste die Politik verstehen, was die Gesellschaft wiederum unter „Tierschutz“ und „Tierwohl“ versteht. So heißt es auch im Strategiepapier der Deutschen Agrarforschungsallianz: „Es soll ein Indikatoren System entwickelt und etabliert werden, mit dem sich ein aussagekräftiges Gesamtbild über den jeweiligen Zustand der deutschen Nutztierhaltung ermitteln lässt. [...] Die Indikatoren müssen an den gesellschaftlichen Zielen anknüpfen [...]“. ${ }^{1}$ Wenn die Bundesregierung wirklich in dieser Legislaturperiode eine staatliche Kennzeichnung etablieren will, braucht sie Informationen über den gesellschaftlichen Wertewandel in der Mensch-Tier-Beziehung. Die bisherigen Ergebnisse des SocialLab sind dafür hilfreich, aber lange nicht ausreichend. Es gibt viel Nachholbedarf. Wie wir als Gesellschaft mit Tieren umgehen wollen, ist keine wissenschaftliche Frage, sondern eine, die pluralistisch in mühsamen Debatten und auf Grundlage bestimmter Werte erschlossen werden muss. Wie der "Stall der Zukunft" aussehen soll (Stichwort: 'Konsumenten bevorzugen Weidehaltung vor Spaltenböden', im Beitrag Kühl et al.), ist dabei längst nicht mehr die einzige Frage. Insbesondere die junge Generation fragt sich: „Sollten (wollen) wir überhaupt Tiere essen? Sollten (wollen) wir überhaupt Milch trinken?“ Die Herausforderung bei der Meinungsforschung besteht darin, den Diskurs nicht so zu lenken, wie man ihn gerne hätte. Das beinhaltet auch, nicht nur wirtschaftlich ,praktikable“ Meinungen zuzulassen. Die Systemfrage der Tierhaltung ist in der Mitte der Gesellschaft angekommen, und sie muss ernst genommen werden. Wenn sich die Agrarpolitik vor unbequemen gesellschaftlichen Anliegen verschließt, werden die Konflikte weiter zunehmen.

\footnotetext{
${ }^{1}$ DAFA (2012) Fachforum Nutztiere. http://www.dafa.de/fileadmin/ dam_uploads/images/Fachforen/Brosch-DAFA-FFNutztiereWeb.pdf. Abgerufen 19.10.2017.
} 